\title{
Variable Endoscopist performance in proximal and distal adenoma detection during colonoscopy: a retrospective cohort study
}

Paul James ${ }^{1,2,3^{*}+}$, Mehdi Hegagi ${ }^{1+}$, Mae Hegagi ${ }^{1}$, Lilia Antonova ${ }^{2}$, Alaa Rostom ${ }^{1,2}$, Catherine Dube ${ }^{1,2}$, Sanjay Murthy ${ }^{1,2}$, Rakesh Goel ${ }^{1}$ and Avijit Chatterjee ${ }^{1,2}$

\begin{abstract}
Background: Adenoma Detection Rate (ADR) is a validated colonoscopy quality indicator. In addition to overall ADR, Distal and Proximal Adenoma Detection Rates may provide important colonoscopy quality information. The goal of this study is to determine the association between distal and proximal adenoma detection (AD) and to identify factors contributing to overall, distal, and proximal AD.

Methods: This is a retrospective cohort study of patients with a noted family history of CRC or positive fecal occult blood test who underwent a screening colonoscopy at a regional colorectal cancer (CRC) screening center between May 2009 and December 2011. Data regarding patient demographics, procedure details, endoscopist characteristics and polyp histology were captured. The main outcomes measured were overall, distal, and proximal AD.

Results: 1907 patients were included. The median age was 60 years and 42\% were male. Endoscopist median overall ADR was 25\% (30\% male, 21\% female). Endoscopist distal ADR was only modestly associated with their proximal ADR (Spearman Rank: $0.51 p=0.11$ ). Highest overall ADR (29 to 45\%) was found for endoscopists whose distal and proximal ADRs were above the group median. In multivariate analysis, factors associated with overall, distal, and proximal AD included age, sex, and endoscopist practicing experience.
\end{abstract}

Conclusion: Inclusion of distal and proximal ADRs, in addition to overall ADR, in colonoscopy quality assessment provides the more accurate feedback on endoscopist performance.

Keywords: Adenoma detection rate, Adenoma, Colonoscopy, Colorectal cancer

\section{Background}

Colonoscopy is considered the most effective method for the identification and removal of adenomatous polyps [1]. However, multiple studies have shown significant polyp and adenoma miss rates for colonoscopies [2], as well as lower than expected reduction in mortality for proximal colorectal cancers [3]. These factors have highlighted the necessity for effective quality indicators to monitor and advance colon cancer screening programs.

\footnotetext{
* Correspondence: paul.james@uhn.ca

${ }^{\dagger}$ Paul James and Mehdi Hegagi contributed equally to this work.

'Department of Medicine, The University of Ottawa, Ottawa, Canada

2Ottawa Hospital Research Institute, The University of Ottawa, Ottawa,

Canada

Full list of author information is available at the end of the article
}

The quality indicator currently recommended by the American Society for Gastrointestinal Endoscopy is Adenoma Detection Rate (ADR) $[4,5]$. ADR is defined as the proportion of colonoscopies where at least one adenoma is found [6]. An endoscopist's ADR has been shown to be associated with overall patient risk of interval colorectal cancer (colorectal cancer diagnosed within a few years of colonoscopy) $[7,8]$, risk of distal interval colorectal cancer [9], and risk of fatal interval colorectal cancer [8].

Little is known about the rate of adenoma detection in specific segments of the colon. Lesions in the distal and proximal colon have been shown to adhere to differing patterns of development. Specifically, sessile serrated adenomas are primarily observed in the proximal colon.

(c) The Author(s). 2018 Open Access This article is distributed under the terms of the Creative Commons Attribution 4.0 International License (http://creativecommons.org/licenses/by/4.0/), which permits unrestricted use, distribution, and reproduction in any medium, provided you give appropriate credit to the original author(s) and the source, provide a link to the Creative Commons license, and indicate if changes were made. The Creative Commons Public Domain Dedication waiver (http://creativecommons.org/publicdomain/zero/1.0/) applies to the data made available in this article, unless otherwise stated. 
These lesions are more difficult to detect and resect and have been proposed to follow a unique serrated pathway that progresses to carcinogenesis more rapidly than the APC-linked pathway of other adenomas [10-12]. Whether this variablity in adenoma presentation in different segments of the colon is reflected in variable rates of adenoma detection between segments, remains to be determined. Recent publications and guidelines have focused on identifying adenomas in the proximal colon. To our knowledge, only two studies to date have examined adenoma detection rates in the distal colon $[13,14]$. These have produced inconsistent results. Whereas Boroff and colleagues observed a lower inter-operator (between endoscopists) ADR for the distal colon, as compared to the proximal colon [13], Schramm and colleagues observed higher distal ADR [14]. Intra-operator (for the same endoscopist) differences for distal and proximal detection have not been previously assessed.

Identifying variability in an endoscopist's detection of distal vs proximal adenomas offers an opportunity to enhance performance assessment and improve colonoscopy quality. The aims of the study presented here are to 1) determine if intra-operator differences exist in distal and proximal ADR and 2) identify factors that predict overall, distal, and proximal adenoma detection.

\section{Methods}

\section{Ethics approval}

This retrospective study protocol was approved by the Ottawa Hospital Research Ethics Board.

\section{Study protocol}

A retrospective study was performed for all high definition white light colonoscopies between May 2009 and December 2011 at the Ottawa Hospital Colorectal Cancer
Screening Clinic - a regional colon cancer screening center. Patients between 50 and 75 years of age, who were referred to the Colon Cancer Screening Clinic with a first degree relative with colon cancer or a positive fecal occult blood test (FOBT), were included. Colonoscopy Interim Reporting Tool (CIRT) forms were mandatorily filled out by the endoscopist of the screening clinic after each procedure. In these forms, procedure indication options included: symptoms, surveillance, positive FOBT, family history of colon cancer in a first-degree relative, and other. The indications of interest in this study included having a family history in a first-degree relative and a positive FOBT. Withdrawal time was not captured. Colonoscopies performed for other indications, including symptoms and colon cancer surveillance, were excluded. Cases where the endoscopist performed less than 50 screening colonoscopies during the study period were also excluded (Fig. 1).

Using the information from the CIRT data collection forms and patient chart review, a database was created containing patient (age and sex), endoscopist (specialty and number of years in practice), and procedure (indication for colonoscopy, bowel preparation, number of polyps found, and the location of each polyp in the colon) information. All bowel preparations involved $4 \mathrm{~L}$ of polyethylene glycol 3350 (Colyte). In the majority of cases, $2 \mathrm{~L}$ split prep dosing was used at the discretion of the endoscopist. Bowel preparation was defined as good when all residue was liquid and over $90 \%$ of the mucosal surface was visible, fair when there was some semisolid stool that could be suctioned or washed away and over $90 \%$ of the mucosa surface was visualized, and poor when semisolid stool could not be suctioned or washed away and less than $90 \%$ of the mucosal surface was visualized [15]. Cases involving good and fair bowel preparation were grouped together, as

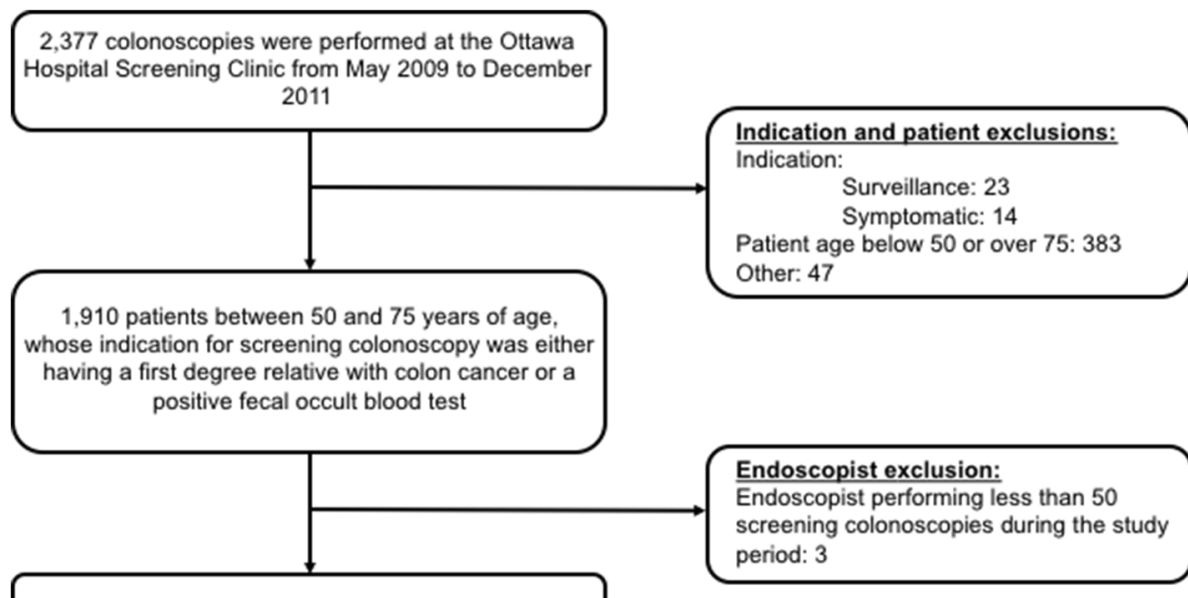

Total number of included cases: 1,907 cases

Fig. 1 Data Inclusions Flowchart 
they have been shown to be associated with similar levels of adenoma detection [15].

Pathology reports were reviewed to determine histology of each colonic polyp removed. Consistent with previous studies [16], all polyps with adenoma or sessile serrated pathology were included as adenomas.

At our center, colonoscopy procedures are scheduled into 30-min time slots. To account for cases where 30 min may have been insufficient time to remove all of the polyps identified during the initial colonoscopy, we determined if an additional colonoscopy was performed within 12 months of the first (index) screening colonoscopy with the use of the Ottawa Hospital Colonoscopy Database. If additional colonoscopies were found, the number, location and histology of each polyp removed within one year of the index colonoscopy were collected and pooled for each patient. We also used this database to calculate each endoscopist's colonoscopy case volume for the period of October 1st 2011 to March 31st 2012.

Each endoscopist was contacted directly in order to obtain information regarding their practicing specialty and their completion of training. This was then corroborated using the College of Physicians and Surgeons of Ontario's public register.

The distribution of colonic polyps was assessed using the following definitions:

Distal colon = splenic flexure, descending colon, sigmoid, and rectum.

Proximal colon $=$ cecum, ascending colon, hepatic flexure, and transverse colon.

$\mathrm{ADR}=$ proportion of colonoscopies where at least one adenoma is found based on histological analysis.
Distal $\mathrm{ADR}=$ proportion of colonoscopies where at least one adenoma is found in the distal colon.

Proximal ADR = proportion of colonoscopies where at least one adenoma is found in the proximal colon.

Adenoma to polyp detection rate quotient $(\mathrm{ADPRQ})=$ proportion of removed polyps found to be adenomas on histological analysis.

\section{Statistical analyses}

Categorical variables were reported as frequencies and proportions while continuous variables were presented as medians with interquartile range. Scatter plot and Spearman rank correlation were used to evaluate the association between ADR, distal ADR, and proximal ADR. Logistic regression models were developed to study univariate and multivariate associations between the independent variables and overall, distal, and proximal AD. Only those variables found to have a significant univariate association ( $p$-value cut-off of $<0.05$ ) with the dependent variables were included in the multivariate models. The data was analyzed using STATA statistical software version 13.1 (StataCorp, College Station, Texas, 2014).

\section{Results}

A total of 1907 patients underwent screening colonoscopies and 11 endoscopists were included in the study (Table 1). The indication for the majority of patients was having a first degree relative with colon cancer (63\%). The mean age was 60 years old and $42 \%$ of patients were male. In most cases (83\%) the endoscopist was a gastroenterologist. Very few of the procedures involved poor bowel preparation (1\%). The cecum was intubated in

Table 1 Cohort demographics and procedure details for the entire cohort, $n=1907$, and patients found to have at least one adenoma on colonoscopy

\begin{tabular}{|c|c|c|c|c|}
\hline & $\begin{array}{l}\text { Total Cohort } \\
(N=1907)\end{array}$ & $\begin{array}{l}\text { Min. one adenoma } \\
(N=467)\end{array}$ & $\begin{array}{l}\text { Min. one proximal adenoma } \\
(N=248)\end{array}$ & $\begin{array}{l}\text { Min. one distal adenoma } \\
(N=308)\end{array}$ \\
\hline Age, Median (IQR) years & $60(55-65)$ & $61(56-66)$ & $62(56-67)$ & $61(55-66)$ \\
\hline Male Sex, $n(\%)$ & $769(41.7)$ & $240(51.4)$ & $128(51.6)$ & $144(52.0)$ \\
\hline \multicolumn{5}{|l|}{ Indication, n(\%) } \\
\hline Family history & $1198(62.8)$ & $263(56.3)$ & $148(59.68)$ & $138(49.8)$ \\
\hline Positive FOBT & 709 (37.2) & $204(43.7)$ & $100(40.3)$ & $139(50.2)$ \\
\hline Poor Bowel Preparation, n(\%) & $27(1.4)$ & $5(1.1)$ & $3(1.2)$ & $2(0.7)$ \\
\hline $\begin{array}{l}\text { Colonoscopy Volumea, } \\
\text { Median (IQR) procedures }\end{array}$ & $150(53-215)$ & $166(96-215)$ & $174(147-215)$ & $157(89-215)$ \\
\hline Cecal intubation, $n(\%)$ & $1857(97.4)$ & $559(98.3)$ & $244(98.4)$ & $273(98.6)$ \\
\hline $\begin{array}{l}\text { Endoscopist Years of Practice, } \\
\text { Median (IQR) years }\end{array}$ & $26(22-38)$ & $23(9-38)$ & $21(9-32)$ & $24(9-38)$ \\
\hline \multicolumn{5}{|l|}{ Endoscopist Specialty, $n(\%)$} \\
\hline Gastroenterology & $1580(83.0)$ & $356(76.2)$ & $212(76.5)$ & $181(73.0)$ \\
\hline Surgery & $327(17.2)$ & $111(23.8)$ & $65(23.5)$ & $67(27.0)$ \\
\hline
\end{tabular}

${ }^{a}$ Case volume over a 6 month period (October 1st 2011 to March 31st 2012) Note: $I Q R$ interquartile range 
almost all of the cases (97\%). More than one colonoscopy was performed within 12 months of the index colonoscopy in 70 patients (4\%). Patient demographics and procedure details are presented in Table 1.

\section{Adenoma detection}

The overall ADR, distal ADR, and proximal ADRs were $25 \%$ (30\% for males and $21 \%$ for females), 15 , and $13 \%$, respectively. The overall adenoma to polyp detection rate quotient (APDRQ) was 50\%. Distal ADPRQ was $38 \%$ and proximal APDRQ was $63 \%$. The median ADR, distal ADR, and proximal ADRs were 28\% (interquartile range [IQR] 20-30\%), 15\% (IQR 11-20\%), and 15\% (IQR 10-19\%), respectively.

\section{Proximal, distal, and overall inter-endoscopist ADR}

Figure 2 presents the overall ADR for each endoscopist as well as the proportion of their ADR that is attributed to adenoma detection in the proximal and distal colon. There was notable variability in both proximal and distal adenoma detection among endoscopists. Distal adenoma detection remained inconsistent even among higher performance endoscopists with overall ADRs greater than $25 \%$.

\section{Correlation between endoscopist overall, distal, and proximal ADR}

Intra-operator differences in distal vs proximal ADR were observed. Two of the endoscopists who had above the median proximal ADR, had below the median distal ADR, while the opposite was observed for two of the other higher performance endoscopists. The highest overall ADR (ADR range 29 to 45\%) was found for the four endoscopists whose distal and proximal ADRs were above the median. The Spearman rank correlation (S) between distal and proximal ADR was $0.51(P=0.11)$, suggesting only a moderate positive association (Fig. 3). Overall ADR was found to be strongly associated with both distal $(\mathrm{S}=0.78, p<0.01)$ and proximal $\mathrm{ADR}(\mathrm{S}=0.83$, $p<0.01)$.

\section{Factors associated with adenoma detection}

Univariate logistic regression (Table 2) showed that overall adenoma detection was significantly associated with patient age (1.03 [95\% CI: 1.01-1.05]), sex (1.68 [95\% CI: 1.36-2.07]), endoscopist specialty (1.77 [95\% CI: $1.37-$ 2.29]) and years of practice (0.97 [95\% CI: 0.97-0.98]). Distal and proximal adenoma detection were also significantly associated with these factors. Endoscopist colonoscopy volume was associated with overall and proximal, but not distal adenoma detection. Having a positive FOBT was associated with overall and distal, but not proximal adenoma detection (Table 2).

In multivariate analyses, age, sex, and endoscopist years of practice remained significantly associated with overall, distal, and proximal adenoma detection. While increased age and male sex were associated with higher adenoma detection, greater endoscopist years of practice was related to lower adenoma detection. The association between adenoma detection and endoscopist specialty was no longer significant (Table 3).

\section{Discussion}

In this study, we demonstrate a notable variability in endoscopist distal and proximal adenoma detection. Our findings suggest that distal and proximal ADR, used in conjunction with overall ADR, can improve the assessment of colonoscopy quality.

The observed variability in adenoma detection rates between the distal and proximal colon may be attributable

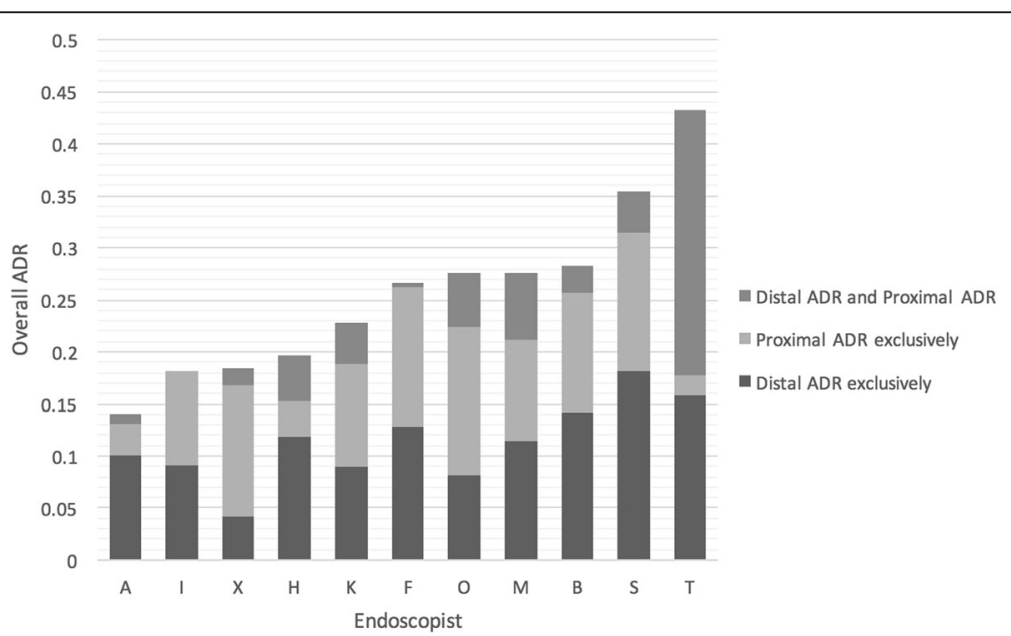

Fig. 2 Adenoma Detection of Each Endoscopist. *Proximal ADR Exclusively = cases where ADR is solely made up of proximal ADR. Distal ADR Exclusively $=$ cases where ADR is solely made up distal ADR 


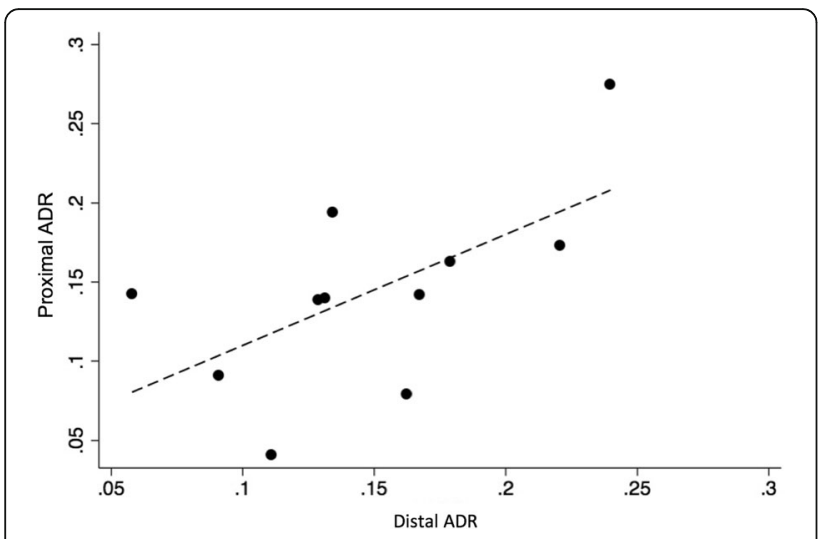

Fig. 3 The association between proximal ADR and distal ADR

to both physiological differences between colon segments and the varibility in operator skill sets. For instance, there are reported differences in segment-specific adenoma occurrence rate $[13,17]$. Boroff and colleagues found that while only $30 \%$ of distal polyps are diagnosed as adenomas on histology, adenomas correspond to $76 \%$ of polyps in the proximal colon [13]. Consistent with these findings, we observed markedly lower distal ADPRQ, as compared to proximal APDRQ (38 and 63\%, respectively).

Adenoma detection is also dependent on skill, training and experience, as demonstrated by significant variability in ADR between endoscopists [18]. Interestingly, adenoma miss rates between endoscopists have been previously found to differ for the distal and proximal colon, suggesting that different skills may be required for these segments [12]. In accordance with this observation, our study demonstrates that proficiency in distal adenoma detection does not directly correlate with proximal adenoma detection for the same endoscopist. This observation was independent of endoscopist overall performance (as indicated by overall ADR). Section-specific operator skills necessary for adenoma detection may relate to variances in polyp morphology [19], frequency [10], and the extent of blind spots in certain parts of the distal and proximal colon [20].

Some specific operator skill measures found to be associated with overall ADR include endoscopist accreditation and colonoscopy volume [21], as well as years of experience [22]. Here we confirm that overall ADR is correlated with all of these operator characteristics.

Table 2 Univariate Analysis for Predictors of Overall, Proximal, and Distal

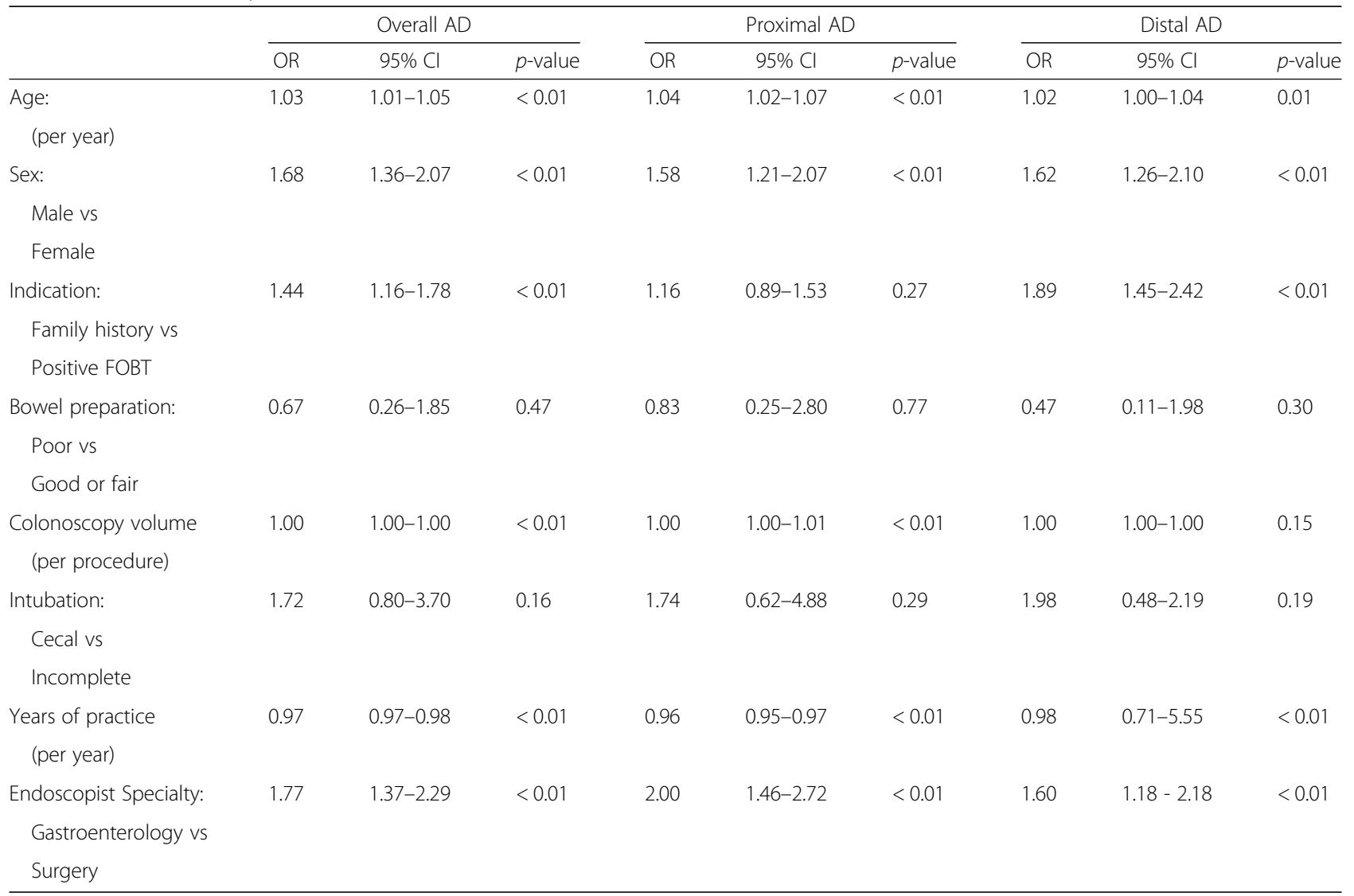


Table 3 Multivariate Analysis for Predictors of Overall, Proximal and Distal

\begin{tabular}{|c|c|c|c|}
\hline & \multicolumn{3}{|c|}{ Overall AD } \\
\hline & $\overline{\mathrm{OR}}$ & $95 \% \mathrm{Cl}$ & $p$-value \\
\hline $\begin{array}{l}\text { Age } \\
\text { (per year) }\end{array}$ & 1.03 & $1.02-1.05$ & $<0.01$ \\
\hline $\begin{array}{l}\text { Sex } \\
\text { Male vs } \\
\text { Female }\end{array}$ & 1.59 & $1.29-1.95$ & $<0.01$ \\
\hline $\begin{array}{l}\text { Indication } \\
\text { Family history vs } \\
\text { Positive FOBT }\end{array}$ & 1.31 & $1.06-1.63$ & 0.01 \\
\hline $\begin{array}{l}\text { Colonoscopy volume } \\
\text { (per procedure) }\end{array}$ & 1.00 & $1.00-1.00$ & $<0.01$ \\
\hline $\begin{array}{l}\text { Years of practice } \\
\text { (per year) }\end{array}$ & 0.98 & $0.97-0.99$ & $<0.01$ \\
\hline \multirow{3}{*}{$\begin{array}{l}\text { Endoscopist Specialty } \\
\text { Gastroenterology vs } \\
\text { Surgery }\end{array}$} & 1.26 & $0.90-1.76$ & 0.18 \\
\hline & \multicolumn{3}{|c|}{ Proximal AD } \\
\hline & OR & $95 \% \mathrm{Cl}$ & $p$-value \\
\hline $\begin{array}{l}\text { Age } \\
\quad \text { (per year) }\end{array}$ & 1.05 & $1.03-1.07$ & $<0.01$ \\
\hline $\begin{array}{l}\text { Sex } \\
\text { Male vs } \\
\text { Female }\end{array}$ & 1.65 & $1.25-2.17$ & $<0.01$ \\
\hline $\begin{array}{l}\text { Colonoscopy volume } \\
\text { (per procedure) }\end{array}$ & 1.01 & $1.00-1.01$ & $<0.01$ \\
\hline $\begin{array}{l}\text { Years of practice } \\
\text { (per year) }\end{array}$ & 0.96 & $0.95-0.98$ & $<0.01$ \\
\hline \multirow{3}{*}{$\begin{array}{l}\text { Endoscopist Specialty } \\
\text { Gastroenterology vs } \\
\text { Surgery }\end{array}$} & 1.13 & $0.73-1.74$ & 0.58 \\
\hline & \multicolumn{3}{|c|}{ Distal AD } \\
\hline & OR & $95 \% \mathrm{Cl}$ & p-value \\
\hline $\begin{array}{l}\text { Age } \\
\quad \text { (per year) }\end{array}$ & 1.02 & $1.00-1.04$ & 0.03 \\
\hline $\begin{array}{l}\text { Sex } \\
\text { Male vs } \\
\text { Female }\end{array}$ & 1.52 & $1.17-1.97$ & $<0.01$ \\
\hline $\begin{array}{l}\text { Indication } \\
\text { Family history vs } \\
\text { Positive FOBT }\end{array}$ & 1.68 & $1.30-2.18$ & $<0.01$ \\
\hline $\begin{array}{l}\text { Years of practice } \\
\text { (per year) }\end{array}$ & 0.99 & $0.97-1.00$ & 0.035 \\
\hline $\begin{array}{l}\text { Endoscopist Specialty } \\
\text { Gastroenterology vs } \\
\text { Surgery }\end{array}$ & 1.15 & $0.77-1.74$ & 0.49 \\
\hline
\end{tabular}

However, while proximal adenoma detection was associated with endoscopist procedure volume, that was not the case for distal ADR. This indicates that the increase in ADR among higher volume endoscopists may be related to their ability to detect adenomas in the proximal colon. Endoscopists with a higher degree of skill and experience are more likely to complete the colonoscopy to the cecum and are better attuned to detecting sessile serrated adenomas [23]. In the setting of limited endoscopy time, if more time is attributed to identifying and removing polyps the proximal colon, the withdrawal time for the distal colon may be compromised, resulting in reduced distal adenoma detection [24].

Adenoma detection also showed a weak inverse association with endoscopy years of practice. This is consistent with previous research demonstrating that optimal ADR occurs with a lower number of years of experience [15], suggesting that training plays a more important role in the quality of adenoma detection than years of experience [25].

Patient-related overall ADR determinants are known to include patient age and gender [26]. Our findings confirm these observations and demonstrate similar associations for both distal and proximal ADR.

Certain study limitations should be noted. First, the study was carried out in a single institution setting. Future investigations should determine if our observations are applicable elsewhere. On the other hand, our observed variability in proximal and distal adenoma detection is consistent with what has been previously observed [14]. Second, we were unable to examine certain procedural factors that have been previously associated with ADR such as withdrawal time [27] and time of day [28, 29]. The influence of colonoscopy queue position during the day, insertion time, and withdrawal time on proximal and distal adenoma detection warrant further study. Finally, we did not differentiate between sessile and non-sessile adenomas. Sessile polyps have been shown to be more difficult to detect and may represent higher risk lesions [30, 31]. However, our approach is consistent with the existing endoscopy quality literature, which includes sessile serrated polyps as adenomas [15]. Finally, the overall ADR for this study was $25 \%$ which, although within the acceptable quality standards for ADR recommended by the American Society for Gastrointestinal Endocopy and the American College of Gastroenterology [1, 5], are lower than ADRs presented elsewhere. We believe that the two main factors contributed our observed lower ADR: the high number of years in practice and low median annual volume of the practicing endoscopists. Both of these factors were associated with lower ADR in this study. This may limit the generalizability of our results and future research should determine whether our findings are reproducible in other centres.

For future investigations, we also recommend the analysis of advanced technologies such as EndoCuff, EndoRings, G-EYE ${ }^{\mathrm{Tm}}$, FUSE, and cap-assisted colonoscopy. These techniques have been related to ADR rates of up 69\%. It has been proposed that using these devices to flatten folds and improving endoscope stability can lead to narrowing 
the adenoma detection differences between the distal and proximal colon [32], however these technologies may be more applicable to augmenting ADR is specific areas of the colon. It has been previously demonstrated that feedback regarding overall ADR results in improvement in proximal but not distal adenoma detection [33]. An additional question of interest would be whether colon segment-specific performance feedback to colonoscopists can produce a more even distribution of improvement.

\section{Conclusions}

We found notable variability in endoscopists' distal and proximal adenoma detection. While distal ADR and proximal ADR are both associated with increased overall ADR, endoscopist distal ADR was only moderately correlated with their proximal ADR. Information regarding both distal ADR and proximal ADR may complement currently used quality indicators to provide endoscopists with informative feedback regarding their adenoma detection proficiency.

\section{Abbreviations}

ADR: Adenoma Detection Rate; APDRQ: Polyp detection rate quotient: CIRT: Colonoscopy Interim Reporting Tool; CRC: Colorectal Cancer; FOBT: Fecal occult blood test; S: Spearman rank correlation

\section{Funding}

1) Patient Quality and Safety Grant, Department of Medicine, Ottawa Hospital

2) Department of Medicine Research Development Grant, Department of Medicine, Ottawa Hospital

The funding bodies did not participate in the study design, data collection, data analysis, results interpretation or writing of the manuscript.

\section{Availability of data and materials}

Datasets used and analyzed during the study are available upon reasonable request from the corresponding author.

\section{Authors' contributions}

PJ, MeH, and AC designed the study. MeH and MaH collected the data. PJ, $\mathrm{MaH}$, and LA interpreted the data. PJ analyzed the data. PJ, MeH, MaH, LA, $A R, C D, S M, R G$, and $A C$ contributed to drafting the manuscript. PJ, MeH, $\mathrm{MaH}$, and LA revised the manuscript. All authors revised and approved the final manuscript.

\section{Ethics approval and consent to participate}

This retrospective study protocol was approved by the Ottawa Hospital Research Ethics Board (institution review board registration number IRB00002616). Need for patient consent to participate was waived.

\section{Competing interests}

The authors declare that they have no competing interests.

\section{Publisher's Note}

Springer Nature remains neutral with regard to jurisdictional claims in published maps and institutional affiliations.

\section{Author details}

'Department of Medicine, The University of Ottawa, Ottawa, Canada. 2Ottawa Hospital Research Institute, The University of Ottawa, Ottawa, Canada. ${ }^{3}$ Department of Medicine, University Health Network, University of Toronto, 200 Elizabeth Street, Room 9N-981, Toronto, ON M5G 2C4, Canada.
Received: 1 July 2017 Accepted: 15 May 2018

Published online: 30 May 2018

\section{References}

1. Rex DK, Johnson DA, Lieberman DA, et al. Colorectal cancer prevention 2000: screening recommendations of the American College of Gastroenterology. American College of Gastroenterology Am J Gastroenterol. 2000;95(4):868-77.

2. van Rijn JC, Reitsma JB, Stoker J, et al. Polyp miss rate determined by tandem colonoscopy: a systematic review. Am J Gastroenterol. 2006;101(2):343-50.

3. Brenner $\mathrm{H}$, Stock $\mathrm{C}$, Hoffmeister M. Effect of screening sigmoidoscopy and screening colonoscopy on colorectal cancer incidence and mortality: systematic review and meta-analysis of randomised controlled trials and observational studies. BMJ. 2014;348:92467.

4. Millan MS, Gross P, Manilich E, et al. Adenoma detection rate: the real indicator of quality in colonoscopy. Dis Colon rectum. 2008;51(8):1217-20.

5. Rex DK, Petrini JL, Baron TH, et al. Quality indicators for colonoscopy. Am J Gastroenterol. 2006:101(4):873-85.

6. Rex DK, Bond JH, Winawer $\mathrm{S}$, et al. Quality in the technical performance of colonoscopy and the continuous quality improvement process for colonoscopy: recommendations of the U.S. multi-society task force on colorectal Cancer. Am J Gastroenterol. 2002;97(6):1296-308.

7. Kaminski MF, Regula J, Kraszewska E, et al. Quality indicators for colonoscopy and the risk of interval cancer. N Engl J Med. 2010;362(19):1795-803.

8. Corley DA, Jensen CD, Marks AR, et al. Adenoma detection rate and risk of colorectal cancer and death. N Engl J Med. 2014;370(14):1298-306.

9. Rogal SS, Pinsky PF, Schoen RE. Relationship between detection of adenomas by flexible sigmoidoscopy and interval distal colorectal cancer. Clin Gastroenterol Hepatol. 2013;11(1):73-8.

10. Rex DK, Ahnen DJ, Baron JA, et al. Serrated lesions of the colorectum: review and recommendations from an expert panel. Am J Gastroenterol. 2012;107(9):1315-29. quiz 4, 30

11. Baxter N, Rabeneck L. New findings about the risks and limitations of colonoscopy used in the early detection of colorectal cancer. Healthc Q. 2009;12(2):24-5.

12. Laiyemo AO, Doubeni $C$, Sanderson AK, et al. Likelihood of missed and recurrent adenomas in the proximal versus the distal colon. Gastrointest Endosc. 2011;74(2):253-61.

13. Boroff ES, Gurudu SR, Hentz JG, et al. Polyp and adenoma detection rates in the proximal and distal colon. Am J Gastroenterol. 2013;108(6):993-9.

14. Schramm C, Mbaya N, Franklin J, et al. Patient- and procedure- related factors affecting proximal and distal detection rates or polyps and adenomas: results from1603 screening colonoscopies. Int J Color Dis. 2015;30(15):1715-22.

15. Clark BT, Rustagi T, Laine L. What level of bowel prep quality requires early. repeat colonoscopy: systematic review and meta-analysis of the impact of preparation quality on. adenoma detection rate. Am J Gastroenterol. 2014;109(11):1714-23. quiz 1724

16. Hilsden, R.J., Dube, C., Heitman, S. J., et al., The association of colonoscopy quality indicators with the detection of screen-relevant lesions, adverse events, and postcolonoscopy cancers in an asymptomatic Canadian colorectal cancer screening population. Gastrointest. Endosc, 2015;82(5):887-894.

17. Caldarella A, Crocetti E, Messerini $L$, et al. Trends in colorectal incidence by anatomic subsite from 1985 to 2005: a population-based study. Int J Color Dis. 2013:28(5):637-41.

18. Anderson JC, Butterly LF. Colonoscopy: Quality Indicators. Clinical and Translational Gastroenterology. 2015;6:e77.

19. Marks JDB. Principles of flexible endoscopy for surgeons. New York: Springer; 2013.

20. Rex DK. Colonoscopy: the current king of the hill in the USA. Dig Dis Sci. 2015:60(3):639-46.

21. Bhangu A, Bowley DM, Horner R, et al. Volume and accreditation, but not specialty, affect quality standards in colonoscopy. Br J Surg. 2012;99(10):1436-44.

22. Mehta MDD, Odstrcil E. Impact of endoscopist's cumulative years of experience on baseline adenoma detection rates and additional yield utilizing the third eye retroscope. Gastrointest Endosc. 2010:71(5):S1597.

23. Jover R, Zapater P, Bujanda $L$, et al. Endoscopist characteristics that influence the quality of colonoscopy. Endoscopy. 2016:48(3):241-7.

24. Barclay RL, Vicari JJ, Doughty AS, et al. Colonoscopic withdrawal times and adenoma detection during screening colonoscopy. N Engl J Med. 2006;355(24):2533-41. 
25. Adler A, Wegscheider K, Lieberman $D$, et al. Factors determining the quality of screening colonoscopy: a prospective study on adenoma detection rates, from 12,134 examinations (berlin colonoscopy project 3, BECOP-3). Gut. 2013:62(2):236-41.

26. Cai B, Liu Z. Xu Y, et al. adenoma detection rate in 41,010 patients from Southwest China. Oncol Lett. 2015;9(5):2073-7.

27. Overholt BF, Brooks-Belli L, Grace M, Rankin K, et al. Withdrawal times and associated factors in colonoscopy: a quality assurance multicenter assessment. J Clin Gastroenterol. 2010;44(4):e80-6.

28. Yeong Teng T, Nan Khor S, Kailasam M, et al. Morning colonoscopies are associated with improved adenoma detection rates. Surg Endosc. 2016;30(5):1796-803.

29. Subramanian S, Psarelli PE, Collins P, et al. Colonscopy performance is stable during the course of an extended three-session working day. Ednosc Int Open. 2015:3(5):E494-500.

30. Macaron C, Vu HT, Lopez R, et al. Risk of Metachronous polyps in individuals with serrated polyps. Dis Colon rectum. 2015:58(8):762-8.

31. Lu Fl, van Niekerk de W, Owen D, et al. Longitudinal outcome study of sessile serrated adenomas of the colorectum: an increased risk for subsequent right-sided colorectal carcinoma. Am J Surg Pathol. 2010;34(7):927-34.

32. Ishaq S, Siau K, Harrison E, et al. Technological advances for improving adenoma detection rates: the changing face of colonoscopy. Dig Liver Dis. 2017:49(7):721-7. Suppl

33. Gawron ATW, Kho A, Keswani R. Improvement in adenoma detection rate after distribution of a report card is due solely to improved detection of right-sided polyps. AGA Abstracts. 2014;146(5):S-730.

Ready to submit your research? Choose BMC and benefit from:

- fast, convenient online submission

- thorough peer review by experienced researchers in your field

- rapid publication on acceptance

- support for research data, including large and complex data types

- gold Open Access which fosters wider collaboration and increased citations

- maximum visibility for your research: over $100 \mathrm{M}$ website views per year

At BMC, research is always in progress.

Learn more biomedcentral.com/submissions 